\begin{tabular}{|c|c|}
\hline Title & The Theory of the Light-Induced Evolution of Hydrogen at Semiconductor Electrodes \\
\hline Author(s) & Bockris, J. O 'M.; Uosaki, K. \\
\hline Citation & $\begin{array}{l}\text { Journal of The Electrochemical Society, 125(2), 223-227 } \\
\text { https://doi.org/10.1149/1.2131418 }\end{array}$ \\
\hline Issue Date & 1978 \\
\hline Doc URL & http:/hdl.handle.net/2115/50260 \\
\hline Rights & $\begin{array}{l}\text { () The Electrochemical Society, Inc. 1978. All rights reserved. Except as provided under U.S. copyright law, this work } \\
\text { may not be reproduced, resold, distributed, or modified without the express permission of The Electrochemical Society } \\
\text { (ECS). The archival version of this work was published in J. Electrochem. Soc. } 1978 \text { volume 125, issue 2, 223-227. }\end{array}$ \\
\hline Type & article \\
\hline File Information & JES125-2_223-227.pdf \\
\hline
\end{tabular}

Instructions for use 


\section{The Theory of the Light-Induced Evolution of Hydrogen at Semiconductor Electrodes}

J. O'M. Bockris and K. Uosaki

J. Electrochem. Soc. 1978, Volume 125, Issue 2, Pages 223-227. doi: $10.1149 / 1.2131418$

Email alerting Receive free email alerts when new articles cite this article - sign up in service the box at the top right corner of the article or click here

To subscribe to Journal of The Electrochemical Society go to: http://jes.ecsdl.org/subscriptions

(C) 1978 ECS - The Electrochemical Society 


\title{
The Theory of the Light-Induced Evolution of Hydrogen at Semiconductor Electrodes
}

\author{
J. O'M. Bockris* and K. Uosaki ${ }^{1}$ \\ School of Physical Sciences, Flinders University of South Australia, Adelaide, Australia
}

\begin{abstract}
The photoelectrode kinetics of the hydrogen evolution reaction is considered, using the WKB approximation for the penetration of the barrier at the semiconductor-solution interface. The absorption characteristics of photons in the electrode are introduced and the number of electrons produced at the surface is obtained as a function of the semiconductor statistics, and also diffusion and field effects. The model makes use of the conclusion that the photo-produced electrons have been deactivated to the bottom of the conduction band by the time they have diffused from the point of photon absorption to the surface. Image energy and the potential difference in the double layer at the semiconductor-solution interface are taken into account. The expression obtained for the photo hydrogen current density is tested in its ability to predict the photo-current-potential curves at the gallium phosphide cathode. Agreement with experiment is fair. Discrepancies are discussed.
\end{abstract}

Although photoeffects on electrochemical reactions at semiconductor-solution interfaces have been studied intensively (1-3), few theoretical analyses have been given $(4-6)$. These all have the substantial defect that they consider the activation of electrons arising from interactions within the semiconductor and neglect an analysis of transfer through the electric double layer at the semiconductor-solution interface.

Photoeffects on electron transfer reactions at the metal/solution interfaces have been studied by Brodsky et al. (7). Bockris et al. (8) have treated photoeffects in hydrogen evolution reaction at metals, using the WKB approximation for electron tunneling through the double layer $(9,10)$.

In the present paper, we apply this approach to photoeffects in the hydrogen evolution reaction at p-type semiconductors, taking into account the activation and transport of photogenerated electrons to the electrode surface. The approach made is quasiphenomenological and does not attempt a general solution, independent of any assumption as to a ratedetermining step.

\section{Assumptions}

In the absence of evidence to the contrary $(11,12)$, it is assumed that charge-transfer is the rate-determining step for the semiconductors used

$$
\mathrm{p}-\mathrm{SC}(e)+\mathrm{H}_{3} \mathrm{O}^{+} \rightarrow \mathrm{p}-\mathrm{SC}-\mathrm{H}-\mathrm{H}_{2} \mathrm{O}
$$

The cathodic current, $i_{c}$, is given by $(8,13)$

$$
i_{\mathrm{c}}=e_{\mathrm{o}} \frac{C_{\mathrm{A}}}{C_{\mathrm{T}}} \int_{0}^{\infty} N(E) W(E) G(E) d E
$$

where $e_{0}$ is the unit charge; $N(E)$ is the number of electrons arriving at the surface per unit area per unit time with energy, $E ; W(E)$ is the WKB tunneling probability of electrons through the potential barrier at energy, $E ; G(E)$ is the distribution function of the vibrational-rotational states of an acceptor, $\mathrm{H}_{3} \mathrm{O}^{+}$, at energy, $E ; C_{\mathrm{A}}$ is the number of acceptors per unit area in the Outer Helmholtz Plane (OHP); $C_{\mathrm{T}}$ is the total number of sites per unit area in the

- Electrochemical Society Active Member.

I Present address: Mitsubishi Petrochemical Company Limited, Ami, Ibaraki, Japan.

Key words: hydrogen, semiconductor, photoeffect, energy level.
OHP. Energy levels are counted as zero in value at the bottom of the conduction band.

In p-type electrodes, the absorption of photons activates electrons in the conduction band where they are available for cathodic reactions. (Contrast thermal electrochemical reactions at the semiconductor-solution interface, where p-type electrodes usually function as anodes).

The validity of the use of the WKB approximation was examined by Sen and Bockris (9), who compared the approach with that of the time-dependent perturbation theory, the results showing that the WKB approximation does not differ in order of magnitude from a time-dependent perturbation calculation for electron transfer (though there are significant discrepancies for proton transfer calculations).

\section{Photon Absorption and Electron Excitation}

The number of photons, the energy of which is $h_{\nu}$, absorbed by the semiconductor between $x$ and $x+d x$ from the surface, $N_{\mathrm{ph}}(x) d x$, is given by

$$
N_{\mathrm{ph}}(x) d x=I_{0}\left(1-R_{\lambda}\right) \alpha_{\lambda} e^{-\alpha_{\lambda} x} d x
$$

where $I_{0}$ is the total number of photons of incident light of energy $h_{\nu}$ per unit area per unit time $\left(\mathrm{cm}^{-2}\right.$. $\left.\mathrm{sec}^{-1}\right) ; R_{\lambda}$ and $\alpha_{\lambda}$ are, respectively, the reflectivity and the absorption coefficient of the semiconductor for the wavelength $\lambda$.

Each absorbed photon, the energy of which is greater than the energy gap of the semiconductor, makes an excited electron in the conduction band and a hole in the valence band. Therefore, the number of electrons excited between $x$ and $x+d x, N_{\mathrm{e}}(x) d x$, is equal to the number of photons absorbed between $x$ and $x+d x, N_{\mathrm{ph}}(x) d x$, and also to the number of holes produced in the valency band. From Eq. [3], $N_{\mathrm{e}}(x) d x$ is given by

$$
N_{\mathrm{e}}(x) d x=N_{\mathrm{h}}(x) d x=N_{\mathrm{ph}}(x) d x
$$

\section{Number of Electrons Arriving at the Electrode Surface}

The electric field at the surface of a semiconductor is well known from the work of Kingston and Neustädter (14). It is possible to extend their result in finding an expression for the field at any point within the semiconductor. 
One finds mean free path for an electron-phonon collision is

$\left(\frac{d V}{d x}\right)_{x}= \pm \sqrt{\frac{8 \pi k T}{e}\left[-\left(N_{D}-N_{A}\right) y+p_{0}\left(e^{-y}-1\right)+n_{0}\left(e^{y}-1\right)\right]}$

where

$$
y=\frac{e_{o}\left(V_{x}-V_{b}\right)}{k T}
$$

and the + sign is for $y<0$.

The number of photoexcited electrons, originally expressed for $x=x$ in Eq. [3] and [4], decreases to $N_{e, x=x-d x}(x) d x$, after traveling $d x$. Then

$$
N_{e, x=x-d x}(x) d x=N_{e}(x) e^{-\frac{1}{L(x)} d x} d x
$$

where $L(x)$, the mean free path of electrons at $x$, is given by (15)

$$
L(x)=\frac{2 l_{D^{2}}}{\sqrt{l_{\mathrm{E}^{2}}+4 l_{\mathrm{D}}^{2}-l_{\mathrm{E}}}}
$$

where $l_{\mathrm{D}}$ is the diffusion length and $l_{\mathrm{E}}$ is the drift length. The terms $l_{D}$ and $l_{E}$ are given by $^{2}$

$$
\begin{gathered}
l_{\mathrm{D}}=\sqrt{\mathrm{D} \tau_{e}}=\sqrt{300 \mu_{e} \tau_{e} k T / e_{\mathrm{o}}} \\
l_{\mathrm{E}}=\tau_{e \mu_{e}} V^{\prime}(x)
\end{gathered}
$$

respectively, where $\mu_{\mathrm{e}}$ is the mobility of the electron $\mathrm{cm}^{2} \mathrm{~V}^{-1} \mathrm{sec}^{-1}, \tau_{e}$ is the lifetime of the electron, and $V^{\prime}(x)$ is the potential gradient at $x\left(-(d V / d x)_{x}\right.$ in $V$ $\mathrm{cm}^{-1}$ ).

Similarly

$$
\begin{gathered}
N_{e_{s} x=x-2 d x}(x) d x=N_{e_{x} x=x-d x}(x) e^{-\frac{1}{L(x-d x)} d x} d x \\
=N_{e}(x) e^{-\left(\frac{1}{L(x)}+\frac{1}{L(x-d x)}\right) d x} d x
\end{gathered}
$$

After $N$ steps $(N=x / d x)$, at the surface

$$
\begin{aligned}
& N_{e, x=0}(x) d x=N_{e, x=d x} e^{-\frac{1}{L(d x)} d x} d x \\
& =N_{e}(x) e^{-\left(\frac{1}{L(x)}+\frac{1}{L(x-d x)}+\cdots+\frac{1}{L(d x)}\right) d x} d x
\end{aligned}
$$

$N_{e, x=0}(x) d x$ is the number of electrons excited between $x$ and $x+d x$ arriving at the surface per unit area per unit time. Therefore, the total number of electrons arriving at the surface per unit area per unit time $N_{\mathrm{e}}\left(h_{v}, V\right)$, is given by

$$
N_{e}\left(h_{\nu}, V\right)=\int_{0}^{\infty} N_{e, x=0}(x) d x
$$

which represents all the electrons excited by photons at any distance inside the semiconductor and which reach the surface, where $V$ is the p.d. inside the semiconductor.

\section{The Energy of an Electron Arriving at the Surface}

The experimentally observed independence of the so-called critical potential (i.e., the potential at which, for light of a given wavelength, the current begins) with the energy of the exciting photons, Fig. 1 (16), suggests that the energies of all the photoexcited electrons are the same by the time they reach the surface. It seems reasonable to postulate that this energy is the bottom of the conduction band. Thus, the average path length of an electron is $1 / \alpha \bumpeq 10^{-4} \mathrm{~cm}$. A typical

$q k$ and $e_{0}$ must be in gaussian units.

(17) $60 \AA$. Hence, a typical energy loss for electrons in reaching the surface is $\left(10^{4 / 60}\right) .0 .025 \mathrm{eV}(\bumpeq 4 \mathrm{eV})$. Thus, the photoexcited electron is effectively deactivated to the energy at the bottom of the conduction band before it reaches the surface (though it does not cross the energy gap, which would need a deactivating cause equivalent in energy to several electron volts).

\section{Electron Transfer Process}

Energy level of an acceptor in solution.-The enthalpy change for electron transfer from a semiconductor to $\mathrm{H}_{3} \mathrm{O}^{+}$. The standard enthalpy change, $\Delta H(e)$, for an electron transfer reaction corresponding to Eq. [1] from the bottom of the conduction band of the semiconductor at the surface to the proton in solution when the proton-solvent system is in its ground state and no potential drop it in the electric double layer can be obtained by using the following thermodynamic cycle

$\mathrm{p}-\mathrm{SC}(e)+\mathrm{H}_{3} \mathrm{O}+$

$$
\uparrow L_{0}
$$

p-SC $(e)+\mathrm{H}^{+}+\mathrm{H}_{2} \mathrm{O}$

$$
\uparrow-E_{\mathrm{a}}
$$

$\mathrm{p}-\mathrm{SC}+e_{(\text {vac })}+\mathrm{H}^{+}+\mathrm{H}_{2} \mathrm{O}$

$$
\begin{array}{cc}
\Delta H(e) \\
& \text { p-SC- }-\mathrm{H}-\mathrm{H}_{2} \mathrm{O} \\
\downarrow-R \\
J & \mathrm{p}-\mathrm{SC}-\mathrm{H}+\mathrm{H}_{2} \mathrm{O} \\
\downarrow-A
\end{array}
$$

where $R, A, J, E_{\mathrm{a}}$ and $L_{0}$ represent the $\mathrm{H}-\mathrm{H}_{2} \mathrm{O}$ repulsive force, the heat of adsorption of a hydrogen atom on the semiconductor, the ionization potential of the hydrogen atom, the electron affinity of the semiconductor, and the hydration energy of proton, respectively. In respect to $R$ and $A$, these quantities are distance dependent and the distance assumed was that appropriate to their state at neutralization. Therefore

$$
\Delta H(e)=-L_{0}+E_{\mathrm{a}}-J+A+R
$$

The energy level of an electron in the ground state of the $\mathrm{H}_{3} \mathrm{O}+$ ion.-By taking into account the potential drop in the electric double layer at the flatband potential (Fig. 2), the energy level of an electron in the ground state of $\mathrm{H}_{3} \mathrm{O}^{+}$with respect to the bottom of the conduction band at the flatband potential, $\Delta H^{\prime}(e)$, is given by

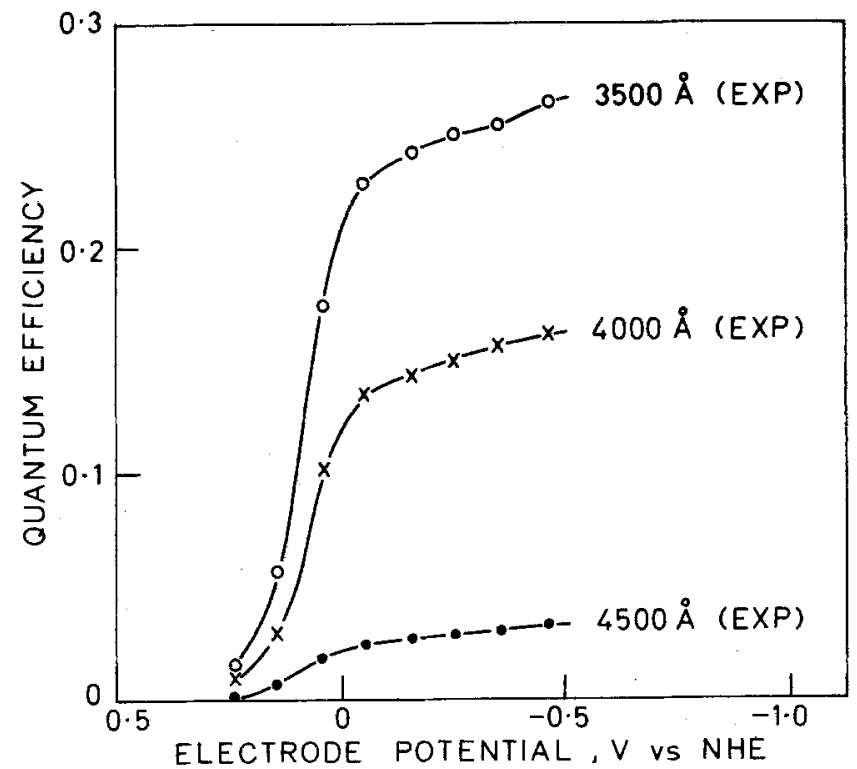

Fig. 1. The experimental quantum efficiency-potential relations of $\mathrm{p}$-GaP in $1 \mathrm{~N} \mathrm{H}_{2} \mathrm{SO}_{4}$. 

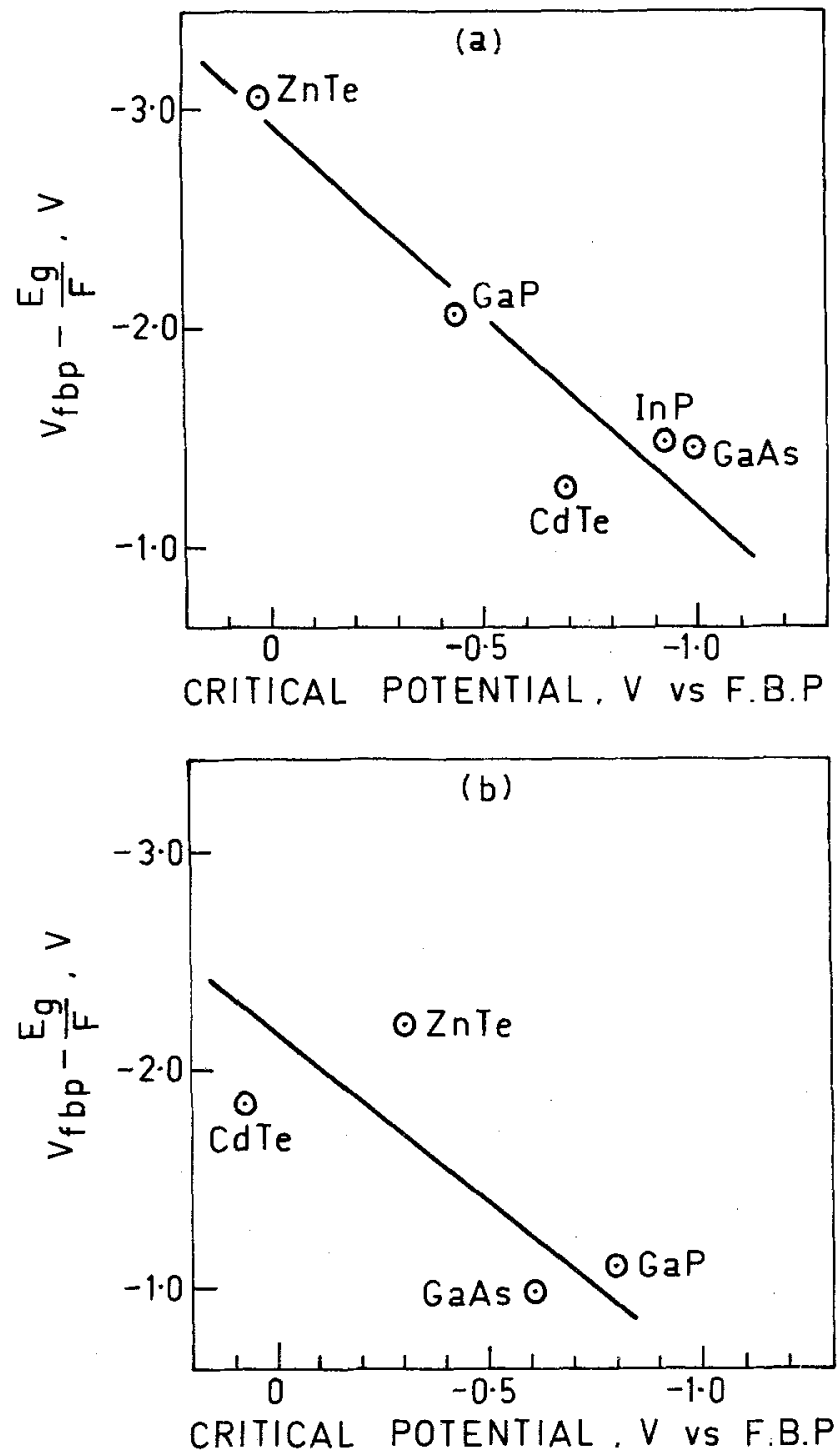

Fig. 2. The relations between $\left(V_{f b p}-E_{g} / F\right)$ and the critical potential with respect to the flatband potential in $1 \mathrm{~N} \mathrm{NoOH}$ (a) and $1 \mathrm{~N} \mathrm{H}_{2} \mathrm{SO}_{4}$ (b).

$$
\begin{aligned}
\Delta H^{\prime}(e) & =\Delta H(e)+F\left(\mathrm{sc}_{\Delta} \mathrm{S}_{\phi}\right)_{\mathrm{fbp}} \\
& =-L_{\mathrm{o}}+E_{\mathrm{a}}-J+A+R+F\left(\mathrm{SC}_{\Delta} \mathrm{S}_{\phi}\right)_{\mathrm{fbp}}
\end{aligned}
$$

where $\operatorname{Sc}_{\Delta} \mathrm{s}_{\phi}$ is an absolute potential $(18,19)$.

The barrier for electron transfer.-Image interaction and the dielectric constant of water.-We have neglected the interaction energy between an emitted electron and its image in a metal in the barrier construction for the photoeffect at metal electrodes (8). In these calculations, the optical dielectric constant was used for the dielectric constant of water. The reason for the neglect of the image interaction was that the emitted electrons travel across the barrier too quickly to make an image in the semiconductor. The optical dielectric constant was used for the same reason.

However, there was some misunderstanding in these considerations. When one wants to construct a potential barrier, one should consider an ideal test charge, i.e., a ("classical") electron moving slowly enough to make its image in the semiconductor. Thus, the interaction energy between the emitted electron and its image should be taken into account in determining the energy barrier and the static dielectric constant of water $[=6$ for the oriented water molecules adsorbed on the electric double layer $(20,21)]$.
The image interaction, $U_{\text {im }}(x)$, is given by

$$
U_{\text {im }}(x)=\frac{e_{0}^{2}}{4 x \epsilon_{\text {st }}} \frac{e_{s t}-e}{\epsilon_{s t}+e}
$$

Interaction with ions in the OHP and their images.When a photoexcited electron leaves the semiconductor surface, it interacts with all ions in the OHP and their electrical images in the semiconductor. The Coulombic force between this electron ( $x$ from electrode) and all ions in OHP and their images, $F(x)$, is given by (8)

$$
\begin{aligned}
F(x)=- & \frac{e_{0}^{2}}{\left(\delta_{\mathrm{r}}-d_{\mathrm{O}-\mathrm{H}}-x\right)^{2} e_{\mathrm{st}}} \\
& +\frac{e_{\mathrm{o}}^{2}}{\left(\delta_{\mathrm{r}}-d_{\mathrm{OH}}+x\right)^{2} \epsilon_{\mathrm{st}}} \frac{-e_{\mathrm{st}}}{\epsilon e_{\mathrm{st}}} \\
- & \frac{2 \pi e_{\mathrm{o}}^{2}}{\epsilon_{\mathrm{st}}} \sum_{n=1}^{\infty}\left\{\frac{d-x}{\left\{(d-x)^{2}+n^{2} R_{1}^{2}\right\}^{3 / 2}}\right. \\
& \left.-\frac{d+x}{\left\{(d+x)^{2}+n^{2} R_{\mathrm{l}}^{2}\right\}^{3 / 2}} \frac{e-\epsilon_{\mathrm{st}}}{\epsilon+\epsilon_{\mathrm{st}}}\right\}
\end{aligned}
$$

where $\delta_{r}$ is the distance between the electrode surface and the nearest proton of the $\mathrm{H}_{3} \mathrm{O}^{+}$ion, $d_{0-\mathrm{H}}$ is the distance between hydrogen and oxygen atom in water, $d$ is the distance between the semiconductor surface and OHP, $\epsilon_{\mathrm{st}}$ is the static dielectric constant of water, $n=1,2,3$ and represents the succession of rings of ions around a given central ion, and $R_{\mathrm{i}}$ is the distance between two ions in the OHP, depending on its coverage with ions and determined by $R_{\mathrm{i}}=4 r_{\mathrm{i}} /(\pi \theta)^{1 / 2}$ where $\theta$ is the coverage and $r_{i}$ is the radius of the ions. Potential barrier.-From the above considerations, the potential energy barrier for electron transfer, $U(x)$, from the surface of the semiconductor surface to the $\mathrm{H}_{3} \mathrm{O}+$ is given by

$$
U(x)=U_{\mathrm{im}}(x)+\int_{0}^{x} F(x) d x+e_{0} X_{x} x
$$

where $X_{x}$ is the field in the double layer.

\section{Potential Drop in the Semiconductor and in the Double Layer}

The potential drop in the electric double layer at the SC-solution interface is often considered to be negligible (22). However, when the carrier density of the semiconductor is high, or the density of the surface states is high, the potential drop in the electric double layer cannot be ignored. We can obtain this quantity from an analysis of the Mott-Schottky plot $(23,24)$.

The Mott-Schottky relation is given by

$$
\frac{1}{C_{\mathrm{SC}^{2}}}=\frac{8 \pi}{\epsilon e_{0} N_{\mathrm{A}}}\left(\psi_{\mathrm{SC}}-\frac{k T}{e_{\mathrm{o}}}\right)
$$

where $C_{S C}$ is the space charge capacity, $N_{\mathrm{A}}$ is the concentration of ionized acceptors, and $\psi_{\mathrm{SC}}$ is the potential drop in the space charge layer.

The appropriate relation in the case of measurements in solution is ${ }^{3}$

$$
\frac{1}{C^{2}}=\frac{8 \pi}{\epsilon e_{0} N_{A}}\left(V-V_{f b p}-\frac{k T}{e_{0}}\right)
$$

where $C$ is the total capacity of the electrode (neglecting the roughness factor) and $V$ and $V_{\text {fbp }}$ are the electrode potential and the fiatband potential with respect to a reference electrode, respectively.

Since (see Fig. 2)

$$
V-V_{\mathrm{fbp}}=\psi_{\mathrm{SC}}+\Delta \Delta \phi_{\mathrm{H}}
$$

${ }^{3} \mathrm{Csc}$ has been assumed equal to $C_{\text {measured because other capaci- }}$ tances (e.g., that of the counterelectrode and Helmholtz layer) are much larger than $C_{s c}$ and hence negligible in series array. 
where

$$
\Delta \Delta \phi_{H}=\left(\operatorname{sc}_{\Delta} \mathrm{s}_{\phi}\right)_{v}-\left(\operatorname{sc}_{\Delta} \mathrm{S}_{\phi}\right)_{\mathrm{fbp}}
$$

and $\Delta \phi$ is the Galvani p.d. in the double layer at $V$ and the $\mathrm{fbp}$, respectively, then, Eq. [21] becomes

$$
\frac{1}{C^{2}}=\frac{8 \pi}{\epsilon e_{0} N_{\mathrm{A}}}\left(\psi_{\mathrm{SC}}+\Delta \Delta \phi \mathrm{H}-\frac{k T}{e_{\mathrm{o}}}\right)
$$

Only when $\Delta \Delta \phi_{\mathrm{H}}=0$ or $\Delta \Delta \phi_{\mathrm{H}}=c \psi_{\mathrm{se}}$, where $c$ is a proportional constant, does the plot between $\left(1 / \mathrm{C}^{2}\right)$ and $V$ become linear $(23,24)$.

If $\Delta \Delta \phi_{\mathrm{H}}=0, \mathrm{Eq}$. [21] becomes Eq. [20] and the slope of the experimental plot (Eq. [21]) must be the same as that of the theoretical plot.

In the case of $\Delta \Delta \phi_{\mathrm{H}}=c \psi_{\mathrm{sc}}$, Eq. [24] becomes

$$
\frac{1}{C^{2}}=\frac{8 \pi}{6 e_{0} N_{A}}\left\{\psi_{\mathrm{sc}}(1+c)-\frac{k T}{e_{0}}\right\}
$$

and thus, $c$ can be calculated by comparing the experimental slope with the theoretical one. This is the case found in recent experimental work (16). $\left(V-V_{\mathrm{fbp}}\right) /(1+c)$ gives the potential drop in the space charge layer in the semiconductor.

De Gryse et al (25) criticized reasoning of this type by showing that the slope of the plot has the same value whether the potential drop occurred in the electric double layer or not. However, in their treatment they assumed an absence of surface charge and this assumption limits the applicability of the interpretation. ${ }^{4}$ The experimental $p H$ dependence of the flatband potential can only be interpreted by assuming the existence of surface states, and the discussion of de Gryse et al. on this matter is no longer directly applicable to situations of this kind.

\section{Photocurrent Expression}

By taking into account the above considerations and Eq. [2], the photocurrent, $i_{p}$, is given by

$$
\begin{aligned}
& i_{\mathrm{p}}= e_{\mathrm{o}} \frac{C_{\mathrm{A}}}{C_{\mathrm{T}}} \int_{0}^{\infty} N(E) W(E) \mathrm{G}(E) d E \\
&= e_{0} \frac{C_{\mathrm{A}}}{C_{\mathrm{T}}} N_{e}\left(h_{\left.\nu, \psi_{\mathrm{SC}}\right)}\right. \\
& \quad \exp \left\{-\frac{\pi^{2} l}{h} \sqrt{2 m_{e}\left(U_{\mathrm{m}}+e_{0} \Delta \Delta \phi_{\mathrm{H}}\right)}\right\} \\
& \quad \exp \left\{-\beta\left(\Delta H(e)+e_{0} \Delta \Delta \phi_{\mathrm{H}}\right) / \mathrm{kT}\right\}
\end{aligned}
$$

where $N_{e}\left(h_{\nu}, \psi_{\mathrm{SC}}\right)$ is the number of electrons arriving at the surface when the potential drop in the semiconductor is $\psi_{\mathrm{SC}}$ and can be obtained from Eq. [13] replacing $V$ by $\psi_{\mathrm{sc}} ; U_{\max }$ is the barrier maximum with respect to the bottom of the conduction band at the flatband potential; $\Delta \Delta \phi_{\mathrm{H}}$ is defined in Eq. [22]; and $V$ is defined in Eq. [21]. The use of $e_{0} \Delta \Delta \phi_{H}$ for the electron energy in the tunneling expression is consistent with a model in which the electrons arrive with a uniform energy at the bottom of the conduction band, i.e., $E_{e}=0$; and the barrier is then influenced by the p.d. in the double layer in the sense that the barrier is reduced when $\Delta \Delta \phi_{H}$ is negative.5,6

\section{Computation of the Photocurrent-Potential Relation and Comparison with Experimental Results}

Photocurrents were calculated for the exampie of $\mathrm{GaP}$ for different wavelengths of light as a function of

In general, the p.d, at a semiconductor-solution interface will be partly in the solution if the coping is very high or there is a significant concentration of surface states.

Equation [25] is no longer an intesral because transfer is seen as occurring only from electrons at the bottom of the conduction and (i.e., photogenerated electrons have been deactivated until hey arrive at the interface at a potential $V_{c}$ )

A rough computation for Gap-NaOH at 0 on the NH scale suggests that about $50 \%$ of the electrons activated by light reach the surface. Those which do not tumnel to the solution deactivate
at the-surface to the valence band.

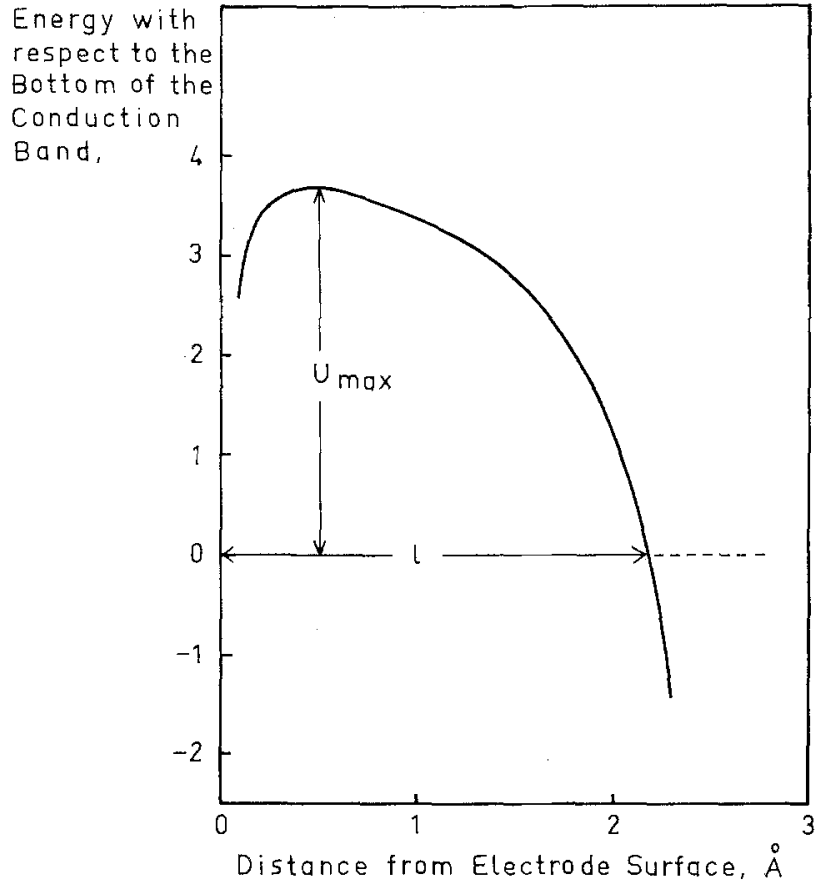

Fig. 3. Schematic diagram of the potential energy barrier for electron transfer from GaP electrode at flatband potential to an acceptor. $\left(E_{\mathrm{g}}\right.$ is the energy gap; critical potential = potential ot which photocurrent commences.)

potential. A schematic energy diagram, which shows the shape of the barrier, is shown in Fig. 3. It was constructed by the use of Eq. [16] and [17] in Eq. [18].

The values used for the calculation of $i_{p}$ through such a barrier by the use of [25] are: $\tau_{e}=10^{-10} \mathrm{sec}$ (26), $\mu_{e}=300 \mathrm{~cm}^{2} / \mathrm{V} \cdot \sec (27), L_{0}=-11.3 \mathrm{eV}(28)$, $E_{\mathrm{a}}=4.3 \mathrm{eV}(27), J=13.6 \mathrm{eV}(28), A=-0.3 \mathrm{eV}$ (29), $R=-0.1 \mathrm{eV}(30), \epsilon=11$ (31), $E_{\mathrm{g}}=2.25 \mathrm{eV}$ (27), $V_{\text {fbp }}=1.13 \mathrm{~V}$ (NHE) (16), and $c=1.32$ (16). $\left(\mathrm{SC}_{\Delta} \mathrm{s}_{\phi}\right)_{\mathrm{fbp}}$ is taken from values given in the paper of Bockris and Uosaki (32). The theoretical results are shown in Fig. 4(a), (b), and (c) and are compared with experimental results. The calculated and experimental results agree fairly in respect to the position and shape of the quantum efficiency-potential relation. The potential at which the photocurrent commences (the so-called critical potential) is predicted to be $0.2-$ 0.4 too positive.

\section{Discussion of Discrepancies Between Theory and Experiment}

Discrepancies between theory and experiment exist as follows:

1. Theoretically estimated quantum efficiencies are only $20-30 \%$ of the experimental quantum efficiencies.

2 . The position of the theoretical quantum efficiency-potential relations appear at about $0.2-0.4 \mathrm{~V}$ more positive than those of experiment.

These discrepancies may be due to cumulative uncertainties in the quantities of Eq. [17] which give the energy levels of electrons in the neutralized $\mathrm{H}_{3} \mathrm{O}^{+}$; $\Delta H(e)$ at the flatband potential and uncertainties in the value of the barrier width, which has been taken at $2.2 \mathrm{~A}$, following the reasoning of Matthews and Bockris (28).

In respect to the determination of $\Delta H(e)$, the difference betwen the Fermi level and the top of the valency band was assumed to be zero. However, this quantity depends on the carrier density and has the order of $0.01 \sim 0.2 \mathrm{eV}$. Were this taken into account, the theoretical quantum efficiency-potential relations would shift toward more negative potentials (i.e., an improvement) by $0.01-0.2 \mathrm{~V}$, depending on the carrier density. 

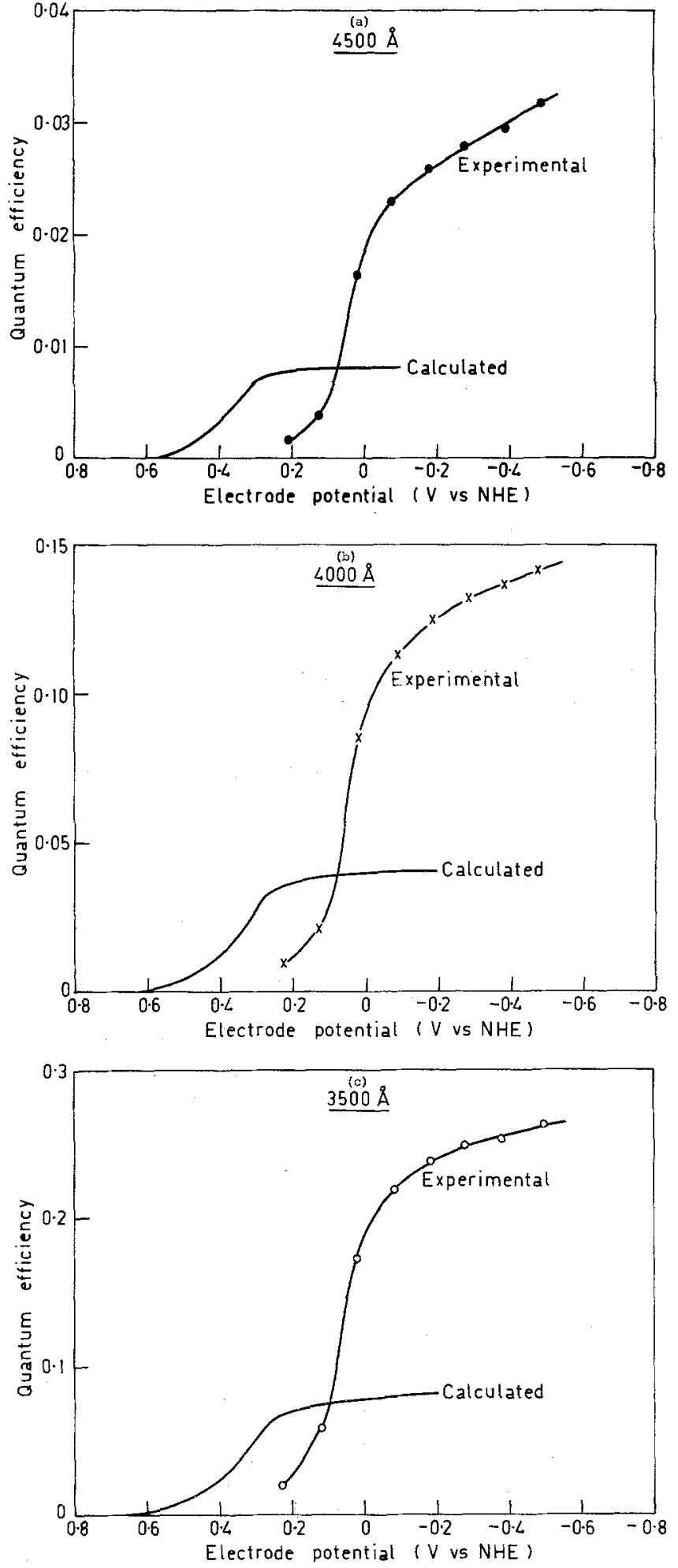

Fig. 4. Calculated and experimental quantum efficiency vs. electrode potential curves for $\mathrm{GaP}$ in $\mathrm{IN} \mathrm{NaOH}$ at the three monochromatic wavelengths (a) 4500; (b) 4000; and (c) 3500 .

In respect to the determination of the barrier dimension, the electron was assumed to transfer to a proton in the second layer of water, to which a proton transfers from $\mathrm{H}_{3} \mathrm{O}^{+}$in the OHP prior to electron transfer (28). ${ }^{7}$ However, it may be possible that a proton transfers to water attached to the electrode surface, and electrons transfer to that. In this case, the barrier thickness becomes much smaller than that assumed and, therefore, higher quantum efficiency

$\tau$ The origin of this model lies in the interpretation of the values observed for the depender barrier widths give separation factors which are too large. than those calculated in the present model would be expected.

\section{Acknowledgment}

We would like to thank Dr. M. A. Habib and Mr. S. U. M. Khan for their useful discussions. One of us (K.U.) thanks Flinders University for a scholarship.

Manuscript submitted Dec. 21, 1976; revised manuscript received Aug. 10, 1977.

Any discussion of this paper will appear in a Discussion Section to be published in the December 1978 JouRNAL. All discussions for the December 1978 Discussion Section should be submitted by Aug. 1, 1978.

Publication costs of this article were assisted by the Flinders University of South Australia.

\section{REFERENCES}

1. H. Gerischer and I. Mattes, Z. Phys. Chem. (N.F.), 49, 112 (1966).

2. Yu. V. Pleskov and V. V. Eletsky, Electrochim. Acta, 12, 707 (1967)

3. R. Memming and G. Schwandt, ibid., 13, 1299 (1968).

4. H. Gerischer, This Journal, 113, 1174 (1966).

5. V. A. Myamlin and Yu. V. Pleskov, "Electrochemistry of Semiconductors," (English ed.), Chap. II Plenum Press, New York (1967).

6. D. Laser and A. J. Bard, Paper submitted to This Journal.

7. A. M. Brodsky and Yu. Ya. Grevich, Electrochim. Acta 13, 1245 (1968).

8. J. O M. Bockris, S. U. M. Khan, and K. Uosaki, J. Res. Inst. Catal., Hokkaido Univ., 24, 1 (1976).

9. R. K. Sen and J. O'M. Bockris, Chem. Phys. Lett., 18, 166 (1973).

10. A. J. Appleby, J. O'M. Bockris, R. K. Sen, and B. E. Conway, in "Physical Chemistry," Vol. 6, J. O'M. Bockris, Editor, MTP Int. Rev. Sci., Butterworths, London (1973).

11. J. O'M. Bockris, S. Srinivasan, and D. B. Matthews, Discuss. Faraday Soc., 39, 239 (1965).

12. M. Enyo, in "Modern Aspects of Electrochemistry," Vol. 11, Chap. 4, J. O'M. Bockris and B. E. Conway, Editors Plenum Press, New York (1975).

13. R. W. Gurney, Proc. R. Soc. London, Ser. A, 134, 137 (1932).

14. R. H. Kingston and S. F. Neustädter, J. Appl. Phys., 26, 718 (1955).

15. S. M. Ryvkin, "Photoelectric Effects in Semiconductors," (English ed.), Chap. XIII, Consultants Bureau, New York (1964).

16. K. Uosaki, Ph.D. Thesis, The Flinders University of South Australia (1976).

17. J. I. Pankove "Optical Processes in Semiconductors," Prentice-Hall, Inc., New Jersey (1971).

18. J. O'M. Bockris and S. Argade, J. Chem. Phys., 49, 5133 (1968).

19. S. Trasatti, J. Electroanal. Chem., 52, 313 (1974).

20. J. O'M. Bockris, M. A. V. Devanathan, and K. Müller, Proc. $R$. Soc, London, Ser. A. 2\%4, 55 (1963).

21. J. O'M. Bockris and M. A. Habib, J. Electroanal. Chem., 65, 473 (1975).

22. F. Möllers and R. Memming, Ber. Bunsenges. Phys. Chem., 76, 469 (1972)

23. T. O. Rouse and J. L. Weininger, This Journal, 113, $184(1966)$.

24. T. P. Biriutseva and Yu. V. Pleskov, Izv. Akad. Nauk. SSSR, Ser. Khim., 251 (1965).

25. R. De Gryse, W. P. Gomes, F. Cardon, and J. Vennik, This Journal, 122, 711 (1975).

26. R. A. Logan and A. G. Chynoweth, J. Appl. Phys., 33, 1649 (1968).

27. "Handbook of Chemistry and Physics," R. C. Weast, Editor, CRC, 55 th ed., E-103 (19749.

28. D. B. Matthews and J. O'M. Bockris, in "Modern Aspects of Electrochemistry," Vol. 6, Chap. 4, J. O'M. Bockris and B. E. Conway, Editors, Plenum Press, New York (1971).

29. J. O'M. Bockris and $K$. Uosaki, Paper submitted to Adv. Chem. Ser

30. R. Schroeder and E. R. Lippincott, J. Phys. Chem., 61, 921 (1959).

31. A. S. Barker, Jr., Phys. Rev., 165, 1674 (1965)

32. J. O'M. Bockris and $K$. Uosaki, Paper submitted to This Journal. 